\title{
18 AEPC Newsletter \\ Paediatric cardiology at the edge of the millennium and quality assessment
}

\author{
Otto Daniëls (Secretary General, \\ Association for European Paediatric Cardiology)
}

\section{$\mathrm{T}$} HE ASSESSMENT OF QUALITY IS NOW A POPULAR topic in the medical profession, and is usually described in the 'buzz phrase' quality assessment. Not only is it the topic of conversation in governmental circles, and amongst those responsible for organisation of health care, but also amongst the professionals working within the profession. And, not least, it has attracted the attention of the patients, who not unreasonably are coming to expect quality in their treatment. It is particularly important that they also should benefit from the result of the assessment of quality. Until now, attention has largely been focussed on requirements for training, for the establishments providing the training, and on continuing medical education. Construction of medical protocols is stimulated. But properly to assess quality, it is also necessary to address the work involved, and the load created in achieving the work. We will take advantage of this issue of our newsletter to summarise the steps taken in the Association for European Paediatric Cardiology to assure the provision of quality, and discuss our intentions for the future.

\section{History}

The Association of European Paediatric Cardiologists was founded in 1961. The founding European paediatric cardiologists, seven in all, started the association with the idea of stimulating scientific research, exchanging clinical experiences, and, by no means least, encouraging good social interchange. So as to maximise the final feature, the association was kept small. Initially, it was permitted for only one paediatric cardiologist in each centre to become a member, although non-mem-

Correspondence to: Otto Däniels, Secretary General, Association for European Paediatric Cardiology, Kinderhartcentrum, Radboud Centraal, Geert Grootplein 10, Postbus 9101, $6500 \mathrm{HB}$ Nijmegen, The Netherlands bers could be invited to the annual congresses. With the passage of time, this policy became oldfashioned. So, in the eighties, it was decided that all paediatric cardiologists could become members providing they had done appropriate scientific work in the field.

In 1990, the 'Treaty of Rome' decreed that there should be free movement of goods and persons throughout Europe. That meant that, in theory, doctors could freely practise medicine in every country of the European Union. For that reason, harmonisation of regulations and rules became mandatory. In the 1980's, therefore, the Association of European Paediatric Cardiologists sought to determine the similarities and differences in the practise of paediatric cardiology within the different countries throughout Europe. At that time, doctors from 24 European countries formed the membership of the Association. Based upon its researches, the Association proposed definitions for the profession, the requirements for training, and the requirements for the institutes providing training. These were accepted by the General Assembly in Oslo, $1990 .{ }^{1}$

By then, it was also clear that the particular area of paediatric cardiology was staffed by physicians originating from two different medical fields: Paediatrics and Cardiology. For that reason, but also for the reason of effectiveness, the Association argued strongly that Paediatric Cardiology should be regarded as a monospecialty. An application for recognition as a monospecialty was therefore submitted to the 'Union of European Monospecialists'. This body, in its wisdom, decided that, since it was clear that Paediatric Cardiology originated from the two fields of Paediatrics and Cardiology, the Association must decide which specialty it wished to join. In the view of the Association, this was an unfair proposal. In effect, the decision had to be made as to whether the child would stay with the father or the mother. After extensive negotiations 
with the specialties of Paediatrics and Cardiology, extending over several years, the Association voted at its Business Meeting in Paris, in 1993, to join Cardiology. The European Specialty for Cardiology made a special arrangement for us. In those deliberations specifically concerned with Paediatric Cardiology, the two representatives of Cardiology in the Union of European Monospecialists would cede their places to two Paediatric Cardiologists nominated by the Association. Paediatric cardiology would then have the right to vote. This was accepted by the Union of European Monospecialists, and Paediatric Cardiology became an Associate Section of this Union. The requirements for training established by the Association were accepted and published in Chapter 6 of the regulations of the Union. ${ }^{2}$ So, in this way the Association changed from a purely scientific organisation to one which was responsible for both scientific and professional affairs.

\section{The Current state of affairs}

It soon became evident that, due to the evolutions in the field, it was necessary to redefine the requirements for training. Moreover, due to expansion of the field, and demands of our colleagues also working in the field of the patient with congenital heart diseases, such as surgeons and morphologists, the Association changed its name, becoming the Association for European Paediatric Cardiology. This association of all medical professionals dealing with the care of the individual with congenital heart disease was essential not least for the fundamental need to stimulate the widest scientific discussion concerning the best treatment for the patients. This could not be achieved when a large part of the profession was barred from membership of the association.

So, at the end of the 1990's, new regulations governing training, and the institutes responsible for providing that training, were drafted in close collaboration with the National Delegates of 25 different countries. In addition, proposals were formulated concerning provision of continuing medical education, re-registration and medical protocols. This process is itself continuous, as it is for all specialties in the medical field.

\section{Consideration}

Despite our best efforts, all these measures are not yet sufficient to guarantee optimal medical care for the individual patient. Requirements for training, and the institutes providing that training are obviously important, as is continuing medical education, but also important are the results of treatment. Governments, companies providing health care, patients, all want to know the results of medical treatment, particularly in respect of figures for mortality and morbidity. Already in countries such as Sweden, the United Kingdom, the Netherlands, and Germany, the authorities, with or without the support of associations of patients, are forcing us to provide these figures. Institutions which are not willing to provide the data are not remunerated for the provision of the treatment! The only organisation capable of providing a proper insight into these complex affairs is the profession itself. So, we have to do it. This means that we have to collect data. In order to achieve this efficiently, we have also to agree on system for diagnostic coding and therapeutic approaches. For this purpose, therefore, the Association has established a Coding Committee made up of acknowledged experts in this field.

Due to these new developments, it is now necessary to construct protocols for medical and surgical treatments. Moreover, it becomes necessary to calculate workloads for the different types of professional activities. For example, we need more insight into the time taken for our medical examinations, the time it costs for our specialists to become involved in other activities, such as teaching, research, management, and so on. To take a specific case, this means that we need to know how many minutes it takes to read an electrocardiogram, and the additional time required to write out its results. Then, knowing the total number of electrocardiograms recorded yearly, we can calculate how much time a department must spend on this item. Only by calculating and summing the time for all activities for a department in this fashion is it possible to assess the total workload. The total workload can then be divided by the number of staff members concerned, and so the workload per person can be calculated. If it can be shown that extreme pressures are imposed by an unreasonable workload, the inference can be made that quality will suffer. Only with the understanding of this problem can a good and deliberate judgement be given over the global field of medical activities. Until now, this aspect seems largely forgotten by the profession. Providing the information will take time, and it will require financing. Yet it is necessary.

We have to convince the authorities, the organisations responsible for health care, and the public, that these measures are inevitable and essential for good provision of medical care. And again, the only body capable of providing these insights is the 
medical profession itself. But, we have to make plain that we are not fighting for our own benefit, but rather for the good of medical care, making 'white magic', care in the benefit of the patient. If this stage is reached, than real assessment of quality comes possible. An inevitable consequence is that the profession will have to accept that it must become increasingly involved in political affairs to achieve these goals.

\section{Summary}

To achieve good and responsible medical care, not only is it necessary to legislate to control training, the institutes responsible for training, and continuing medical education, but it is also necessary to document rating $s$ of mortality and morbidity as well as measures of workload for those providing care. We, as an Association for Paediatric Cardiology, must be more active in this process, and in politics, so as to convince the patients and the authorities that these processes are necessary, and to obtain the financial requirements to permit them to be achieved. Much has still to be done.

\section{References}

1. Daniels O, Choussat A 1994 Paediatric Cardiology in Europe,Medical/ScientificStatement of the Association of European Paediatric Cardiologists. Definition of profession and training requirements. Eur Heart J 1994; 15: 160-163.

2. Chapter 6 'Charter on Training of Medical Specialists'. In the European Union Requirements for the Specialty 'Paediatric Cardiology'. Division of the UEMS Section 'Cardiology'. Union of European Monospecialists, Summary, draft June 1995. http: //www.uems.be/cardped.htm 